\title{
Sectores Estratégicos en la Economía del Conocimiento y Desarrollo en la Ciudad de Madrid y su Región*
}

\section{Strategic Sectors in the Knowledge Economy and Development in the City of Madrid and its Region}

\author{
Simón Sánchez-Moral \\ simon.sanchez@ghis.ucm.es @ https://orcid.org/0000-0001-6381-2653 \\ Roberto Díez-Pisonero \\ rdpisonero@ghis.ucm.es @ https://orcid.org/0000-0002-7817-628X \\ Cándida Gago-García \\ cgago@ucm.es@ https://orcid.org/0000-0003-2315-7943 \\ Universidad Complutense de Madrid. Facultad de Geografía e Historia. \\ c/ Profesor Aranguren s/n. Madrid-28040
}

Alfonso Arellano-Espinar

alfonso.arellano@pdi.ucm.es @ https://orcid.org/0000-0002-1464-8751

Universidad Complutense de Madrid. Facultad de Ciencias Económicas y Empresariales.

Campus de Somosaguas 28223 - Pozuelo de Alarcón (MADRID)

\section{INFO ARTÍCULO}

Recibido: $1 / 5 / 2019$

Revisado: 6/6/2019

Aceptado: 7/6/2019

\section{PALABRAS CLAVE}

Sectores estratégicos

Innovación

Redes empresariales

Estrategias urbanas

\section{KEYWORDS}

Strategic sectors

Innovation

Firm networks

Urban strategies

\begin{abstract}
RESUMEN
Diversas agendas internacionales (OCDE, Europa 2020) recogen la apuesta por las actividades intensivas en conocimiento e innovación. El objetivo de esta investigación es analizar el carácter estratégico de las industrias de alta intensidad tecnológi$\mathrm{ca}$, los servicios intensivos en conocimiento y las industrias culturales en Madrid. Se comienza revisando el planteamiento de los sectores estratégicos en grandes capitales europeas. El análisis de las redes empresariales confirma la importancia de estos sectores en la conexión global de Madrid y en la articulación de sus flujos económicos, pero también lo heterogéneo de las dinámicas temporales y espaciales del empleo. Sin perder de vista la tendencia a replicar la selección sectores estratégicos similares entre las grandes ciudades, su protagonismo en las estrategias de desarrollo urbano y regional estaría sólidamente fundamentado en el caso de Madrid.
\end{abstract}

\begin{abstract}
Different international agendas (OECD, Europe 2020) consider the promotion of knowledge based activities and innovation. The objective of this research is to analyze the strategic role of high technology-intensive industries, knowledge-intensive services and the cultural industries in Madrid. The paper begins by reviewing the approaches to strategic sectors in large European capitals. The analysis of firm networks in the region confirms the importance of these sectors in Madrid's global connections and the articulation of their economic flows, but also the heterogeneous nature of the temporal and spatial trends of employment. Without losing sight of the tendency by large cities to replicate the selection of similar strategic sectors, their role in urban and regional development strategies would have a solid base in the case of Madrid.
\end{abstract}

* Este trabajo se enmarca dentro del proyecto “ Atracción de talento, innovación y creatividad para el desarrollo inteligente de la ciudad-región de Madrid" Ref. CSO2016-74888- C4-4-R (AEI/ FEDER, UE). 


\section{INTRODUCCIÓN: HACIA UN MARCO INTERPRETATIVO SOBRE SECTORES ESTRATÉGICOS Y COMPETITIVIDAD URBANA}

El proceso de globalización y la creciente integración de la economía mundial han favorecido niveles crecientes de competencia entre empresas y entre territorios. En este contexto, las ciudades buscan asegurar la generación de ventajas competitivas dinámicas, las cuales se fundamentarían en una dotación muy destacada de recursos específicos de calidad, más o menos tangibles, como empleo cualificado, servicios avanzados y las infraestructuras de comunicación; pero sobre todo a través de redes de colaboración empresariales y socio-institucionales que impulsan los procesos de innovación (Salom, J. y Albertos, J.M., 2009; Musterd, S. y Murie, A., 2010). Las grandes ciudades verían así reforzada su posición a la cabeza del sistema urbano, especializándose en sectores y funciones de mayor rango, destacando aquellas más intensivas en el uso de tecnología y conocimiento (Scott, A.J., 2008 y 2009; Méndez, R. y Sánchez Moral, S., 2011).

Muchas de estas actividades forman parte de lo que hace décadas Machlup ya identificó como sectores con alta intensidad de conocimiento, relacionados con las tecnologías y los servicios de información, la educación, el I+D, la creación artística o los medios de comunicación (Machlup, F., 1962). También se relaciona con las propuestas de K. Pavitt (1984), quien añadió la perspectiva de la intensidad tecnológica y el tipo de innovaciones predominantes en las distintas actividades. A partir de aquí, distintos organismos internacionales, como UNCTAD, OCDE o Eurostat, han tratado de acotar los límites sectoriales de la llamada "economía del conocimiento", a partir de las clasificaciones de actividades económicas vigentes, como punto de partida para valorar el nivel de implantación y su contribución en el desarrollo de los territorios. Con todo, actualmente suele identificarse un conjunto de clusters innovadores o estratégicos en el contexto del capitalismo cultural- cognitivo (Scott, A.J., 2008) y que aparecerían especialmente desarrollados en las grandes metrópolis: industrias de alta intensidad tecnológica, servicios intensivos en conocimiento, dentro de estos los servicios avanzados a las empresas, así como las industrias culturales (Wu, W., 2005).

En relación con estas propuestas conviene no perder de vista algunas cuestiones importantes. Por un lado, dentro del debate existente se reconoce igualmente la existencia de zonas de solapamiento donde emergen otras actividades y funciones urbanas que podrían considerarse igualmente estratégicas para las grandes ciudades, en relación por ejemplo con el ocio, el consumo o el turismo (Glaeser, E.L., 2011). De hecho, en una aproximación más transversal a la economía del conocimiento, se entiende que el conocimiento (como recurso) y la innovación económica y social (como estrategia) son susceptibles de mejorar el desempeño de cualquier tipo de actividad, favoreciendo en último término el desarrollo urbano (Widen, W., et al., 2007; Méndez, R., 2013).

Por otro lado, desde diferentes foros de pensamiento económico se advierte un interés renovado por la identificación de sectores estratégicos, sabedores del importante papel que estos juegan en la resiliencia territorial y la competitividad urbana y regional, así como de la necesidad de priorizar las acciones de política económica. Dicha identificación está presente en los diferentes enfoques existentes dentro de las políticas de corte sectorial y que algunos autores como W. Warwick (2013) contraponen a otras políticas más horizontales y que persiguen resolver problemas generales, como por ejemplo las políticas de convergencia ("catch-up policies"), destinadas a que los países o regiones alcancen el nivel óptimo y competitivo respecto a otros países. En concreto, dentro de las aproximaciones sectoriales cabría diferenciar entre aquellas que persiguen el conservar una ventaja ya adquirida con respecto a competidores (en relación por ejemplo con sectores maduros) y las que buscan crear "ex novo" fortalezas, afianzando nuevos sectores económicos o sectores de vanguardia ("frontier industrial policies"). En relación con esto último, cabe destacar igualmente el papel de las estrategias de especialización inteligente de la Unión Europea que, aunque con un planteamiento horizontal, también persiguen efectos sobre sectores concretos, relacionados con la innovación y el avance tecnológico a escala regional (Comisión Europea, 2010a y 2010b; Foray, D., et al., 2011).

En este contexto, el objetivo de esta investigación es contrastar el carácter estratégico de las actividades de la economía del conocimiento en el caso de la ciudad- región de Madrid y actualizar el diagnóstico sobre su marcha reciente, ofreciendo con ello un marco de reflexión para orientar las prioridades de la ciudad en 
materia de promoción y ordenación de estas actividades². Esta reflexión parte de una serie de interrogantes iniciales que emanan de la propia revisión de la literatura:

En primer lugar, resulta evidente la apuesta a escala internacional por una economía más intensiva en innovación y conocimiento, con vistas a hacer frente al reto de la competitividad, unido al de la equidad y la sostenibilidad ambiental, lo que exige de nuevas estrategias que mejoren la resiliencia territorial en esta etapa postcrisis (OCDE, 2010a; Comisión Europea, 2010a; Sánchez-Moral, S., 2015). Nuestra hipótesis de trabajo es que esta visión estratégica ha penetrado en las capitales europeas, incluida Madrid. Esto es algo que queremos contrastar en relación con la posible apuesta por otro tipo sectores, que igualmente serían considerados estratégicos.

En segundo lugar, queremos confirmar que efectivamente las estrategias empresariales que dan forma, junto con otros procesos espaciales, a la reestructuración metropolitana en curso (Scott, A.J., 2008; Paddison, R. y Hutton, T., 2015), como por ejemplo las estrategias de descentralización productiva, de internacionalización o de innovación tecnológica, han reforzado el carácter estratégico de las actividades de la economía del conocimiento para Madrid. En particular, nos proponemos analizar el papel de las relaciones input-output que ligan a las actividades de la economía del conocimiento con el resto de la economía regional, contribuyendo a la articulación interna de esta y a mejorar su competitividad en un contexto cada vez más globalizado.

Por último, queremos verificar el grado de implantación de este tipo de actividades en la economía y el territorio de Madrid, que podría estar reforzando su posición dentro del bicéfalo sistema urbano español, al menos desde el punto de vista de la localización de sedes de empresas, servicios avanzados a las empresas o actividades financieras (Taylor, P.J., 2013); al tiempo que Barcelona se posiciona como hub cultural- creativo internacional (Boix, 2011). En todo caso, Madrid se sitúa en principio alejado de los principales centros europeos líderes en la economía del conocimiento (ESPON, 2013). Además, desde el punto de vista del dinamismo reciente, cabe preguntarse por esa capacidad de las actividades intensivas en conocimiento para proteger a las economías regionales ante las crisis económicas y convertirse en un "refugio" para el empleo (Comisión Europea, 2013; Perlo, M., 2011). Al respecto, conviene no perder de vista algunas tendencias no tan positivas, como la segmentación y precarización del empleo, el impacto de la crisis sobre los sistemas regionales de innovación o de las políticas de austeridad sobre las estrategias de innovación e investigación (Jordá, R., et al., 2014). Con todo, cabe relacionar la marcha de la economía del conocimiento con la propia trayectoria y especificidad de los territorios urbanos, comenzando por sus recursos materiales y humanos, la calidad institucional y la gobernanza local (Pratt, A.C., 2012; Méndez, R., 2013; Méndez, R., et al., 2016).

\section{METODOLOGÍA}

La investigación se fundamenta en la siguiente definición de los clusters innovadores de la economía del conocimiento (Wu, W., 2005), obtenida de la revisión de la literatura:

1. Industrias de alta intensidad tecnológica. De acuerdo con la OCDE se trata de actividades con un gasto en I+D por encima del $4 \%$ de su facturación y, además, con una proporción mayor de trabajadores cualificados. Entre las principales actividades cabe mencionar la industria farmacéutica, el material electrónico e informático y la construcción aeronáutica. Estas quedan incluidas dentro de las clasificaciones recientes de EUROSTAT sobre "knowledge intensive industries".

2. Servicios intensivos en conocimiento. La estrecha relación de algunos servicios con esos sectores industriales de alta intensidad tecnológica sirve como punto de partida para la definición de EUROSTAT de servicios intensivos en conocimiento (servicios financieros, telecomunicaciones, educación, sanidad...). La delimitación no está exenta de ciertos problemas relacionados, sobre todo, con su elevada

2. Una primera versión de este diagnóstico tuvo su origen en el contrato "Bases para la identificación de sectores estratégicos en la ciudad de Madrid" (Ayuntamiento de Madrid, 2016). 
heterogeneidad interna y la presencia de algunas ramas con elevado peso de empleo público (Musterd, S., et al., 2007).

3. Dentro del grupo anterior suele identificarse el subconjunto de los Servicios avanzados a las empresas (como consultoría, contabilidad, publicidad, arquitectura,...). Todos ellos tendrían una importancia estratégica desde el punto de vista del valor añadido, la generación y el consumo de innovaciones y la creación de empleo de calidad. Surgidas con frecuencia de procesos de externalización de empresas industriales, estas actividades son también fundamentales desde la perspectiva de la articulación interna y competitividad de las economías urbanas, contribuyendo a generar un continuo de actividades servindustriales. Su elevada productividad les permitiría resistir mejor las tendencias deslocalizadoras hacia territorios con menores costes.

4. Industrias culturales. Por un lado, se alude a aquellas industrias que combinan la creación, producción y comercialización de contenidos que son por su naturaleza intangibles y culturales (UNESCO, 2006). Se parte de la idea que actualmente el capitalismo se sitúa en una fase donde la generación de productos culturales es crucial en la estrategia productiva (Scott, A.J., 2008). El concepto se relaciona también con las llamadas "industrias creativas", que suman a las actividades culturales tradicionales (edición de libros, música, artes escénicas,...), otras como el software de videojuegos, las actividades de arquitectura, el diseño o la publicidad. Estas actividades son importantes impulsoras de la innovación económica y social en otros muchos sectores, siendo con frecuencia el origen de soluciones imaginativas relacionadas, por ejemplo, con la creación de marcas, el desarrollo de capacidades tecnológicas, el aprendizaje permanente o el estímulo a la investigación (Comisión Europea, 2010c).

A partir de aquí, el estudio plantea tres aproximaciones metodológicas complementarias. En primer lugar, se comienza haciendo un repaso a las estrategias emprendidas en los diferentes niveles escalares de la intervención, en relación con la selección y promoción de sectores económicos estratégicos. En concreto, se han analizado nueve ciudades europeas, seleccionadas por ocupar los primeros puestos en rankings de ciudades de carácter económico. Lógicamente, todas ellas son potenciales competidoras de Madrid. La información analizada es de naturaleza diversa, incluyendo: (i) estrategias de desarrollo económico elaboradas por ayuntamientos, gobiernos regionales o ambos, dependiendo del ámbito de competencias que tengan atribuido las diferentes administraciones; (ii) el papel que se otorga en webs institucionales o en documentos de citimarketing de aquellos sectores considerados claves por las oficinas $u$ organismos regionales de promoción económica y de inversión. La principal dificultad a la hora de analizar esta documentación es la variación en la propia denominación de los sectores. En ocasiones estos aparecen agrupados en denominaciones muy genéricas, mientras que en otros casos se especifican subsectores de forma detallada. En este sentido, de forma intencionada no se ha acotado el ámbito sectorial de esta identificación para poder valorar la importancia otorgada a los sectores intensivos en tecnología y conocimiento en el marco de las respectivas economías locales.

En segundo lugar, el estudio se adentra en la importancia de las actividades estratégicas en la articulación del sistema productivo regional y de investigación. Concretamente, de forma complementaria a los estudios de caso habitualmente empleados en investigaciones monográficas que parten de la construcción ad- hoc de indicadores (Navarro Arancegui, M. y Gibaja Martins, J.J., 2009), se propone aquí un sistema de análisis sistemático de los vínculos input-output. En concreto, buscamos evaluar la capacidad de las actividades seleccionadas para inducir dos tipos de efectos. En primer lugar, nos fijamos en los llamados efectos de arrastre entre una actividad económica y aquellas otras que le sirven de proveedor, de tal forma que si el sector económico se reactiva gracias a un shock, sus proveedores experimentan sinergias positivas derivadas de un aumento de la demanda de ese sector. También este efecto se asocia al concepto de eslabonamiento hacia atrás. En segundo lugar, nos fijamos en el llamado efecto impulso entre esa actividad económica y aquellas otras que constituyen sus clientes, de modo que un impacto positivo en el sector económico de estudio repercute favorablemente en su provisión de productos, beneficiando a los compradores de dicho producto. También este efecto se asocia al concepto de eslabonamiento hacia delante. 
Para obtener una cuantificación de estos efectos se ha utilizado la última tabla input-output disponible para la Comunidad de Madrid (TIOCM-2010). En este sentido, el desglose a 66 ramas de actividad no coincide exactamente con la CNAE-2009, por lo que no siempre es posible ajustarse a la definición inicial sobre clusters innovadores. Pese a estas limitaciones técnicas, la tabla input-output permite generar diversos indicadores de interés, siendo necesario hacer tres apuntes sobre la metodología desarrollada, basada en los trabajos de Collado, J.C., et al. (2003):

1. La tabla input-output pone énfasis en las transacciones que tienen lugar entre las diferentes ramas de actividad, ofreciendo una imagen nítida de la economía de un territorio como un sistema de flujos interdependientes, incluyendo sus relaciones con otros territorios (exportaciones e importaciones). Permite registrar el origen (dentro de la economía o exterior a través de la importación) de cada consumo, sea éste de carácter intermedio o final.

2. Los coeficientes técnicos expresan la utilización que cada rama hace de productos de otra rama por unidad de producción. Estos coeficientes miden el impacto directo de una rama sobre otra cuando se produce un aumento de la demanda final de la primera cifrado en una unidad. Los coeficientes técnicos constituyen la base de las interconexiones existentes entre las distintas actividades económicas que constituyen el tejido productivo. Así, sirven de instrumento para medir las interrelaciones sectoriales directas, tanto el eslabonamiento directo hacia atrás como el eslabonamiento directo hacia delante.

3. Por otro lado, los elementos de la matriz inversa de Leontief expresan la cuantía en que debe variar la producción total de una rama de actividad si se desea aumentar en una unidad la demanda final de otra rama de actividad. De esta forma, para captar los efectos directos de empuje y arrastre que una rama genera en otra se utilizan los coeficientes técnicos, mientras que si se quiere captar los efectos directos e indirectos (generando un efecto multiplicador en la economía) se emplean los coeficientes de la matriz inversa de Leontief.

Este análisis se completa con una valoración del papel de las actividades intensivas en tecnología y conocimiento desde la perspectiva de otras interacciones generadas con las instituciones de educación superior e investigación de la región, a través de fuentes indirectas.

Finalmente, se plantea el análisis de las dinámicas temporales y espaciales del empleo afiliado, por medio de los microdatos de la Seguridad Social (2011-2016), obtenido ex profeso para esta investigación. El análisis a nivel de dos dígitos de la CNAE2009 y para los distritos de la ciudad y de los municipios de la región de Madrid (según disponibilidad estadística), permite obtener una imagen detallada tanto del dinamismo reciente, como de las tendencias espaciales, analizadas mediante cartografía basada en el cálculo de los cocientes de localización del empleo.

\section{RESULTADOS Y DISCUSIÓN}

\subsection{Sectores estratégicos en ciudades europeas}

El repaso a las propuestas de aquellos organismos cuya misión fundamental es el diseño de las políticas (OCDE y Unión Europea) confirma que las políticas económicas sectoriales que emanan de su reflexión estratégica tienden a replicarse en los diferentes ámbitos de aplicación; desde la propia Unión Europea, pasando por los Estados miembros y las Administraciones locales. Ello suele llevar a una identificación ciertamente similar de sectores estratégicos intensivos en tecnología y conocimiento.

Por lo que respecta a las nueve capitales europeas analizadas (Londres, París, Berlín, Ámsterdam, Bruselas, Zúrich, Barcelona, Viena, Estocolmo), todas comparten la misión de la promoción económica en las respectivas ciudades, considerando como premisa básica un contexto altamente competitivo entre las grandes ciudades del mundo.

Se observa, no obstante, diferentes planteamientos estratégicos a la hora de identificar los sectores. Así, por ejemplo, algunas estrategias inciden en el concepto de la ventaja tecnológica competitiva ("revealed te- 
chnology advantage"), lo que permitiría a la ciudad continuar especializándose y potenciar determinadas actividades por encima de otras; beneficiándose en primer lugar de las capacidades locales y en segundo lugar de una posición relevante frente a territorios competidores. Otras estrategias inciden en la importancia de las condiciones concretas del entorno empresarial, necesarias para el desarrollo de las actividades productivas. En cuanto a los sectores concretos que se consideran estratégicos, estos están en clara relación con las directrices marcadas por los organismos internacionales. En concreto, cuatro son comunes a las nueve ciudades analizadas, esto es, industrias de la salud y de la vida, industrias creativas y culturales, tecnologías de la información y telecomunicaciones (estos dos últimos deben considerarse en estrecha relación temática y, de hecho, algunos documentos analizados los consideran conjuntamente). Otros cuatro sectores más, el sector financiero, las industrias verdes (fundamentalmente aquellas relacionadas con la producción de energías renovables), la educación superior y el turismo aparecen consideradas también en la mayor parte de los casos analizados (Tabla 1).

En este punto cabe destacar la coherencia en la selección de sectores estratégicos en las ciudades del entorno europeo con el planteamiento observado en las últimas décadas en el caso de Madrid. Habitualmente, los ejercicios de identificación de sectores estratégicos de este tipo han partido del gobierno de la Comunidad de Madrid; por ejemplo, a través de la política de clusters productivos de los años noventa del pasado siglo; o, de forma más reciente, a través de la formulación de su Estrategia de Especialización Inteligente: "Estrategia Regional de Investigación e Innovación 2014-2020 (RIS3)" (Comunidad de Madrid, 2013). En este sentido, RIS3- Madrid plantea cuatro áreas prioritarias en torno a Nanociencia, Materiales Avanzados, Tecnologías Industriales y del Espacio; Energía, Medio ambiente y Transporte (incluida la Aeronáutica); Salud, Biotecnología Agua y Agroalimentación; Tecnologías de la información y comunicaciones (TIC) y Servicios de alto valor añadido. Por su parte, el Ayuntamiento de Madrid propuso en la década pasada una selección de "sectores servindustriales estratégicos", donde además de algunas industrias de alta intensidad tecnológica, como la aeronáutica o la farmacéutica, se ponía el énfasis en las tecnologías de la información y comunicaciones, así como en los servicios avanzados a las empresas (Sánchez Moral, S., 2006).

En suma, la identificación de sectores o clusters estratégicos en ciudades europeas parece responder, por un lado, al planteamiento de las estrategias territoriales de innovación, basado en diagnostico sistemático de las capacidades locales y los nichos de oportunidad, como en el caso de las estrategias de especialización inteligente (Foray, D., et al., 2011). No obstante, se observa al mismo tiempo el impacto de esas "vehicular ideas" o "políticas móviles" que viajan entre regiones, tal como se ha señalado en la literatura especializada (Peck, J., 2012). En este sentido, la tarea de identificación de los sectores estratégicos es crucial teniendo en cuenta la similitud de opciones que consideran los responsables de la Administración. Más aún, la mayor parte de las estrategias analizadas se acompañan de una cohorte de medidas para facilitar las condiciones de instalación y desarrollo de los distintos clusters (dotación de suelo e infraestructura, ventanillas únicas, exenciones fiscales y ayudas a las startups de componente innovador, entre otras). Dichas medidas, que influyen directamente sobre el desempeño final de los mismos (junto con otros factores como la atracción de flujos de capital y de talento, la formación o capacitación del capital humano, muy presentes también en las políticas), tienden igualmente a ser replicadas entre las ciudades analizadas.

\subsection{El papel de los sectores estratégicos en la articulación del sistema productivo y de innovación de la región}

La Tabla 2 sintetiza los resultados obtenidos siguiendo la metodología Collado, J.C., et al. (2003) descrita anteriormente. Esta distingue entre valores por encima y por debajo de la media en los eslabonamientos directos y los efectos totales, tanto para los valores de Madrid como para los valores totales, incluyendo la parte correspondiente a las importaciones. Dado que nuestro objetivo es valorar el papel de las actividades 
Tabla 1. Sectores considerados estratégicos en una selección de ciudades europeas.

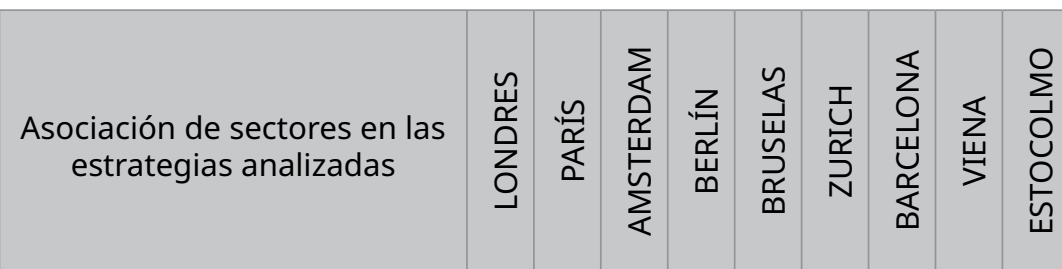

\begin{tabular}{|c|c|c|c|c|c|c|c|c|c|c|}
\hline Industrias de la salud y de la vida & - & & & & & & & & & \\
\hline Industrias creativas y culturales & & & & & & & & & & \\
\hline Turismo & & & & & & & & & & \\
\hline Tecnologías de la información & & & & & & & & & & \\
\hline Telecomunicaciones & & & & & & & & & & \\
\hline Financiero & & & & & & & & & & \\
\hline Servicios avanzados a las empresas & & & & & & & & & & \\
\hline Educación superior & & & & & & & & & & \\
\hline Investigación y desarrollo & Mencionados conjuntamente & & & & & & & & & \\
\hline Industrias verdes (incl. energías renovables) & & & & & & & & & & \\
\hline Movilidad (sostenible) & Mencionados conjuntamente & & & & & & & & & \\
\hline Automóviles & & & & & & & & & & \\
\hline Agricultura y alimentación & - & & & & & & & & & \\
\hline Logística & & & & & & & & & & \\
\hline Aeronáutica/ espacial & 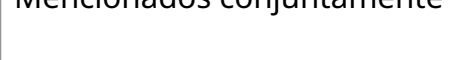 & & & & & & & & & \\
\hline Comercio & - & & & & & & & & & \\
\hline Comercio al por menor & - & & & & & & & & & \\
\hline Micro y nanotecnología & - & & & & & & & & & \\
\hline Electrónica & - & & & & & & & & & \\
\hline Energía & - & & & & & & & & & \\
\hline Óptica (fotónica entre ellas) & - & & & & & & & & & \\
\hline Metal & - & & & & & & & & & \\
\hline Instrumentos & - & & & & & & & & & \\
\hline Materiales de alta tecnología & - & & & & & & & & & \\
\hline Industrias químicas y del plástico & - & & & & & & & & & \\
\hline Mecatrónica & - & & & & & & & & & \\
\hline Número de sectores estratégicos: & - & 10 & 13 & 13 & 17 & 15 & 9 & 13 & 9 & 8 \\
\hline
\end{tabular}

Fuente: Elaboración propia, procedente y adaptado de Ayuntamiento de Madrid (2016). 
Tabla 2. Descomposición de los efectos de las actividades de la economía del conocimiento y del resto de la economía.

\begin{tabular}{|c|c|c|c|c|}
\hline & $A(T)$ & $B(T)$ & $C(T)$ & $\mathrm{D}(\mathrm{T})$ \\
\hline$\sum_{\bar{\alpha}}$ & & $\begin{array}{l}\text { - Agua, saneamiento y } \\
\text { residuos } \\
\text { - Cine, video, TV, radio } \\
\text { y sonido } \\
\text { - Construcción general } \\
\text { - Informática } \\
\text { - Productos metálicos } \\
\text { - Publicidad y estudios } \\
\text { de mercado } \\
\text { - Sedes centrales y } \\
\text { gestión empresarial } \\
\text { - Serv. anexos al trans- } \\
\text { porte } \\
\text { - Serv. técnicos }\end{array}$ & $\begin{array}{l}\text { - Actividades jurídicas } \\
\text { y de contabilidad } \\
\text { - Alquileres } \\
\text { - Transporte terrestre } \\
\text { de mercancías }\end{array}$ & - Artes gráficas \\
\hline$\sum_{\infty}$ & - Metálicas básicas & $\begin{array}{l}\text { - Act. postales y de } \\
\text { correos } \\
\text { - Cemento y derivados } \\
\text { - Cuero y calzado } \\
\text { - Edición } \\
\text { - Ordenadores y ma- } \\
\text { quinaria de precisión } \\
\text { - Otra química final } \\
\text { - Servicios de informa- } \\
\text { ción } \\
\text { - Transporte no terres- } \\
\text { tre }\end{array}$ & - Madera & $\begin{array}{l}\text { - Alojamientos } \\
\text { - Ind. mueble } \\
\text { - Otras act. profesio- } \\
\text { nales } \\
\text { - Restaurantes y bares } \\
\text { - Seguros y fondos de } \\
\text { pensiones } \\
\text { - Serv. recreativos } \\
\text { Serv. personales } \\
\text { - Transp. terrestre de } \\
\text { viajeros }\end{array}$ \\
\hline$\underset{\Sigma}{U}$ & $\begin{array}{l}\text { - Caucho y plástico } \\
\text { Industria del papel }\end{array}$ & $\begin{array}{l}\text { - Comercio y reparación } \\
\text { de vehículos } \\
\text { - Industrias cárnicas } \\
\text { - Maquinaria indus- } \\
\text { trial } \\
\text { - Otras alimenticias } \\
\text { - Otras manufacturas } \\
\text { - Vidrio e industrias no } \\
\text { metálicas } \\
\text { - (Telecomunicaciones) }\end{array}$ & $\begin{array}{l}\text { - Act. Inmobiliarias } \\
\text { - Aux. a construcción } \\
\text { - Comercio mayorista } \\
\text { - Energía eléctrica y gas } \\
\text { - Industrias extractivas } \\
\text { - Intermediación finan- } \\
\text { ciera } \\
\text { - Otras act. administra- } \\
\text { tivas } \\
\text { - (Telecomuniciones) }\end{array}$ & $\begin{array}{l}\text { - Auxiliares financie- } \\
\text { ros y de seguros } \\
\text { - Otro comercio mino- } \\
\text { rista }\end{array}$ \\
\hline$\sum_{0}$ & $\begin{array}{l}\text { - Coquerías y refino de } \\
\text { petróleo } \\
\text { - Química de base e } \\
\text { industrial }\end{array}$ & $\begin{array}{l}\text { - Bebidas y tabaco } \\
\text { - Confección } \\
\text { - Material electrónico } \\
\text { - Material eléctrico } \\
\text { - Otro material de } \\
\text { transporte } \\
\text { - Productos farmacéu- } \\
\text { ticos } \\
\text { - Vehículos y sus } \\
\text { piezas }\end{array}$ & $\begin{array}{l}\text { - Agricultura y gana- } \\
\text { dería } \\
\text { - Industria textil }\end{array}$ & $\begin{array}{l}\text { - Act. asociativas } \\
\text { - Act. hogares } \\
\text { - Admon. públicas } \\
\text { - Educación } \\
\text { - Ind. lácteas } \\
\text { - Sanidad y Servicios } \\
\text { Sociales }\end{array}$ \\
\hline
\end{tabular}

Nota grupos: A: Niveles por encima de media en eslabonamientos directos y efectos totales; B: Niveles por encima de media en eslabonamiento directo hacia atrás y/o el efecto arrastre; C: Niveles por encima de media en eslabonamiento directo hacia delante y/o efecto impulso; D: Niveles por debajo de media en eslabonamientos directos y efectos totales; (T): Datos totales; (M): Datos para la región de Madrid Fuente: Elaboración propia, procedente y adaptado de Ayuntamiento de Madrid (2016). 
intensivas en tecnología y conocimiento en el contexto de la economía regional, el análisis incluye las 66 ramas de las TIO (2010) organizadas en cuatro grupos de sectores ${ }^{3}$ :

- Grupo A: constituido por aquellas ramas de actividad que tienen valores por encima de la media en los eslabonamientos directos hacia atrás y hacia delante, así como en los efectos arrastre e impulso.

- Grupo B: formado por las actividades que presentan niveles por encima de la media en el eslabonamiento directo hacia atrás y/o el efecto arrastre.

- Grupo C: incluye los sectores económicos que disponen de niveles por encima de la media en el eslabonamiento directo hacia delante y/o el efecto impulso.

- Grupo D: incorpora a las ramas de actividad que tienen valores por debajo de la media en los eslabonamientos directos hacia atrás y hacia delante, así como en los efectos arrastre e impulso.

Haciendo referencia a las actividades pertenecientes a los clusters innovadores identificados al comienzo, cabe señalar que las industrias de alta intensidad tecnológica presentan una capacidad de arrastre a nivel global considerable, como consumidoras de inputs intermedios de otras muchas actividades. Esta influencia se extiende hacia delante en el caso concreto de la industria química de base e industrial. Sin embargo, las industrias de alta intensidad tecnológica muestran un papel mucho más discreto cuando se consideran los datos del interior de la región de Madrid, salvo para los ordenadores y maquinaria de precisión, así como la maquinaria industrial, en este caso por generar impulsos hacia delante. En otras palabras, estas actividades sí estarían generando cierto efecto multiplicador sobre empresas de la región. En el resto de actividades el importante grado de internacionalización e integración de las actividades en grupos multinacionales, unido a la tendencia a la reducción de algunos clusters regionales en las últimas décadas (Méndez, R., et al., 2007), explicaría que los principales efectos se dirijan, sobre todo, hacia el exterior de la región.

Frente a esto, la mayoría de servicios avanzados a las empresas (como informática, sedes centrales y gestión empresarial, servicios técnicos, publicidad y estudios de mercado, así como actividades jurídicas y de contabilidad) se posicionan como actividades estratégicas para el funcionamiento y la generación de valor de las empresas de la región. Más aún, la mayor parte de ellas presentan a su vez efectos de arrastre directos o indirectos igualmente notables fuera del territorio regional, salvo algunas excepciones como las actividades jurídicas y de contabilidad (con mayor importancia en los efectos de impulso), así como otras actividades profesionales.

Cabe relacionar todo ello con la mencionada concentración de funciones terciarias avanzadas en Madrid, como núcleo rector del sistema urbano español, a través del que se canalizan y articulan el grueso de los flujos productivos nacionales e internacionales. En efecto, como señala la abundante literatura internacional sobre Knowledge Intensive Business Services (KIBS), la gestión de esos flujos en un contexto de incertidumbre y creciente integración de las regiones y empresas en la globalización sitúa en una posición nuclear a los servicios avanzados a las empresas (Cuadrado Roura, J.R., 2013). Ello queda plenamente confirmado en el caso de la región madrileña.

Por lo que respecta a otras actividades de servicios intensivas en conocimiento, como telecomunicaciones, intermediación financiera o servicios auxiliares financieros y de seguros, estas se perfilan como actividades fundamentales para la expansión del tejido productivo regional. En este sentido, mención especial merece el caso de las telecomunicaciones, que forma parte del Grupo C para el caso de los datos del interior de Madrid. Sin embargo, obtiene resultados diferentes entre los eslabonamientos directos y los efectos totales para los valores globales, registrando un valor por encima de la media en el eslabonamiento directo hacia atrás y también en el efecto impulso.

Por último, las industrias culturales presentan efectos multiplicadores domésticos por encima de la media. En el caso de actividades como cine, video, televisión, radio y sonido estos efectos incluyen además el impulso hacia delante. Salvo en el caso de los servicios recreativos, una parte importante de ese arrastre vía el consumo de inputs intermedios alcanza a actividades fuera de la región. El resultado viene a confirmar el

3. El Anexo I recoge los coeficientes técnicos que fundamentan esta clasificación por grupos (por motivos de espacio se incluye solo el detalle de las actividades relacionadas con la economía del conocimiento). 
carácter estratégico de estas actividades que, junto con otras como publicidad, software, etc., suelen agruparse bajo el epígrafe de la "economía creativa" (DCMS, 1998). Tal como señala la propia Comisión Europea "Ias industrias culturales y creativas representan un conjunto de empresas altamente innovadoras, con un gran potencial económico, y constituyen uno de los sectores más dinámicos en Europa" (Comisión Europea, 2010c, p. 2). Por lo tanto, junto con la contribución al crecimiento y al empleo regional, el caso de Madrid confirma su importante papel de cara a la generación de efectos multiplicadores sobre otras actividades o el impulso a la innovación, económica y social, dentro de los clusters creativos (Cooke, P. y Lazzeretti, L., 2008).

Obviamente existen otros vínculos y formas de interacción de los sectores de la economía del conocimiento con el resto del sector productivo (incluidas otras relaciones de colaboración no de mercado). Profundizar en estos aspectos exige de un estudio monográfico, fuera de los objetivos de esta investigación. Sin embargo, sí parece oportuno mencionar, al menos, la existencia de vínculos que se establecerían dentro del sistema regional de innovación y, en concreto, entre las empresas de las actividades identificadas y los grupos de investigación pertenecientes a instituciones de educación superior y centros de investigación de la región. Para ello nos basamos en los resultados del diagnóstico previo de RIS3- Madrid (Comunidad de Madrid, 2013). De los 333 grupos de investigación entrevistados durante el proceso de diagnóstico, hasta 295 declararon colaborar en proyectos con empresas de sectores relacionados con los aquí seleccionados. Destaca el caso de I+D (113 colaboraciones en proyecto) y los productos farmacéuticos (80), muy por delante del resto de sectores de la economía madrileña; además de la industria química (36), los productos informáticos y electrónicos (30), las telecomunicaciones (20), otras actividades profesionales científicas y técnicas (18), la programación y consultaría informática (12), el transporte aéreo (7) o la arquitectura, ingeniería y ensayos (7). En definitiva, muchas de estas actividades se erigen como elementos fundamentales dentro del Sistema Regional de Innovación de Madrid, caracterizado ya por R. Jordá et al. (2014) como un SRI de excelencia centrados en la alta tecnología.

\subsection{Nivel de desarrollo de los sectores estratégicos en la economía y el territorio madrileño}

La última dimensión del análisis considera el carácter estratégico de las actividades desde el punto de vista de las dinámicas del empleo. Sin perder de vista las diferencias metodológicas entre los estudios y las dificultades a la hora de adscribir las actividades de innovación, creatividad o I+D+i, cabe recordar, en primer lugar, que el empleo en la ciudad de Madrid podría rondar la cuarta parte del empleo total en economía del conocimiento del país (Méndez, 2013). No obstante, el análisis de los datos de empleo y su evolución reciente arroja resultados contrastados por clusters innovadores (por razones de disponibilidad estadística el análisis está limitado a la ciudad de Madrid). En primer lugar, es evidente el desigual peso de estos, destacando por orden de importancia los servicios avanzados a las empresas, con 269.150 empleos en 2016, por delante de otros servicios intensivos en conocimiento, que contaban con 114.894 empleos, las industrias culturales, con 39.484 empleos y las industrias de alta intensidad tecnológica, cuyo volumen se ve reducido hasta los 19.865 empleos. Desde el punto de vista de la importancia en la economía local, cabe señalar que el peso en el empleo local de las industrias de alta intensidad tecnológica resulta muy reducido (ligeramente superior al 1\%). Limitado resulta también el desarrollo del cluster de actividades culturales, siendo su peso en el empleo local del $2,15 \%$. Otros servicios intensivos en conocimiento se mueven en torno al $6 \%$ del total del empleo de la ciudad, mientras que los servicios avanzados a las empresas alcanzan valores del 14,68\% (Tabla 3).

En relación con la capacidad de resiliencia de los clusters innovadores, cabe señalar que el descenso más acusado entre 2011 y 2016 correspondió a la industria de alta intensidad tecnológica (-17,31\%), afectada por los ajustes de capacidad durante la crisis y las decisiones de (des)localización de las multinacionales del sector (Méndez, R., 2013). Igualmente negativo resultó el comportamiento del empleo en las industrias culturales (-13,79\%), en relación sobre todo con el sector de la edición (precisamente la actividad de naturaleza más industrial del cluster). Por el contrario, otros sectores que vivieron un ajuste importante en la fase de la austeridad, como fue el caso de la creación artística, los espectáculos o las actividades de museos y 
archivos, salen en cambio mejor parados en el global del periodo. Por su parte, otros servicios intensivos en conocimiento, que experimentaron una caída menor $(-2,90 \%)$, parecen recuperarse una vez superada la reestructuración del sector financiero y muy especial de la entidad Bankia y de los agentes financieros de su antigua red comercial. Frente a esto, los servicios avanzados a las empresas presentan una tasa de variación positiva del $14 \%$. En este sentido, se confirma su capacidad de recuperación en la salida de la crisis, ya observada en la segunda fase de la misma por R. Méndez et al. (2015), una vez se recupera la demanda por parte del tejido productivo madrileño y la contratación del sector público. En definitiva, las evidencias apuntan a una recuperación del dinamismo del empleo, al menos en parte de la economía del conocimiento.

Tabla 3. Evolución del empleo en clusters estratégicos en la ciudad de Madrid.

\begin{tabular}{|c|c|c|c|c|}
\hline Cod. CNAE-2009 & Actividad & Empleo (2016) & $\begin{array}{l}\% \text { Empleo } \\
\text { Local }\end{array}$ & $\begin{array}{c}\text { Tasa de variación } \\
2011 / 16\end{array}$ \\
\hline 20 & Industria química & 1.895 & 0,10 & $-16,19$ \\
\hline 21 & Fabr. de productos farmacéuticos & 3.747 & 0,20 & $-19,32$ \\
\hline 26 & Prod. informáticos, electrónicos, ópticos & 880 & 0,05 & $-15,79$ \\
\hline 28 & Fabr. de maquinaria y equipo N.C.O.P & 4.238 & 0,23 & $-28,68$ \\
\hline 29 & Fabr. vehículos de motor, remolques... & 6.573 & 0,36 & $-10,49$ \\
\hline \multirow[t]{2}{*}{30} & Fabr. otro material de transporte & 2.532 & 0,14 & $-9,21$ \\
\hline & Total cluster 1 & 19.865 & 1,08 & $-17,31$ \\
\hline 62 & Programación, consultoría informática... & 73.408 & 4,00 & 40,96 \\
\hline 63 & Servicios de información & 7.568 & 0,41 & 5,23 \\
\hline 69 & Actividades jurídicas y de contabilidad & 55.649 & 3,03 & 6,41 \\
\hline 70 & Act. sedes centrales; gestión empresarial & 19.767 & 1,08 & 18,20 \\
\hline 71 & Servicios técnicos de arquitectura... & 47.036 & 2,57 & 1,12 \\
\hline 72 & Investigación y desarrollo & 15.313 & 0,84 & $-2,48$ \\
\hline 73 & Publicidad y estudios de mercado & 32.972 & 1,80 & 1,59 \\
\hline \multirow[t]{2}{*}{74} & Otras act. profesionales, científicas... & 17.437 & 0,95 & 32,85 \\
\hline & Total cluster 2 & 269.150 & 14,68 & 14,00 \\
\hline 61 & Telecomunicaciones & 31.014 & 1,69 & 4,09 \\
\hline 64 & Servicios financieros, exc. seguros... & 48.645 & 2,65 & $-14,17$ \\
\hline 65 & Seguros, reaseguros y fond. pensiones & 18.218 & 0,99 & 6,68 \\
\hline \multirow[t]{2}{*}{66} & Act. auxiliares servicios financieros... & 17.017 & 0,93 & 15,14 \\
\hline & Total cluster 3 & 114.894 & 6,27 & $-2,90$ \\
\hline 58 & Edición & 15.954 & 0,87 & $-29,31$ \\
\hline 59 & Act. cinematográficas, vídeo, prog. TV... & 8.201 & 0,45 & $-5,14$ \\
\hline 60 & Act. programación, emisión radio y TV & 2.406 & 0,13 & $-2,20$ \\
\hline 90 & Act. creación, artísticas y espectáculos & 10.367 & 0,57 & 7,92 \\
\hline \multirow[t]{2}{*}{91} & Act. bibliotecas, archivos, museos... & 2.556 & 0,14 & 1,51 \\
\hline & Total cluster 4 & 39.484 & 2,15 & $-13,79$ \\
\hline
\end{tabular}

Fuente: Elaboración propia, procedente y adaptado de Ayuntamiento de Madrid (2016). 
Para evaluar el grado de implantación de las actividades estratégicas en el territorio de la Comunidad de Madrid se han analizado las tendencias de localización del empleo, agregadas por clusters innovadores. Los municipios que acogen un mayor número de trabajadores en industrias de alta intensidad tecnológica son: Madrid (19.034 empleos, 30,9\% del total), cuyo poder de atracción sobre estas actividades es algo mayor al que tiene sobre la industria manufacturera en general. Junto con ello, destaca el caso de Alcobendas (11.287 empleos, 18,3\%), Getafe (6.407 empleos, 10,4\%), Alcalá de Henares (3.047 empleos, 4,9\%) y Tres Cantos (2.597 empleos, 4,2\%), localizaciones tradicionales todas ellas para este tipo de actividades industriales. Dentro del municipio de Madrid, los distritos con mayor número de empleos en estas actividades son San Blas-Canillejas y Villaverde, ámbitos donde la actividad industrial en general mantiene presencia en algunas de las áreas industriales más consolidadas de la ciudad (Méndez, R., Ondátegui, J. y Sánchez Moral, S., 2007). Estas tendencias quedan perfectamente reflejadas en los niveles de especialización a nivel de municipios de la región y distritos de la capital, que dibujan el conocido arco por el este de la ciudad; desde Colmenar Viejo, Tres Cantos y Alcobendas al norte; pasando por Ajalvir y varios municipios del corredor del Henares, con Alcalá a la cabeza; hasta el sur metropolitano, donde destacan Getafe, Pinto y Fuenlabrada, en conexión con el tejido del sector sur de la ciudad (Figura 1).

En cuanto a los servicios avanzados a las empresas, Madrid es el municipio de la región que acoge el mayor volumen de trabajadores en servicios avanzados a las empresas ( 239.758 empleos, $70,8 \%$ del total), seguido de otros municipios del Norte y Oeste metropolitano, como Alcobendas (16.439 empleos, 4,8\%), Las Rozas de Madrid (12.466 empleos, 3,7\%), Pozuelo de Alarcón (11.725 empleos, 3,4\%) o Tres Cantos (8.514 empleos, 2\%). Todos estos ámbitos aparecen especializados a nivel regional, evidenciándose la importancia que adquiere el norte metropolitano, así como la difusión hacia el oeste metropolitano. En la ciudad destaca el peso y nivel de especialización que se alcanza en varios distritos, como Salamanca, Chamartín, Centro, o San Blas-Canillejas, entre otros. En definitiva, se confirma el fuerte arraigo del terciario avanzado en la mitad norte de la ciudad y en la periferia metropolitana, conformando un tejido económico continuo, al que contribuyen tanto la localización en los espacios de oficinas más centrales de la ciudad, como en los numerosos parques empresariales desarrollados en las últimas décadas en un entorno metropolitano de elevada calidad ambiental y nivel socioeconómico (Méndez, R. y Sánchez Moral, S., 2011).

Por lo que respecta a otras actividades intensivas en conocimiento, estas se concentran preferentemente en la ciudad central (especialmente en el caso de los servicios financieros y las telecomunicaciones). En concreto, Madrid registra el mayor número de trabajadores (94.656 empleos, 73,7\% del total), seguido de otros municipios de nuevo del norte y oeste metropolitano, como Boadilla del Monte (11.071 empleos, 8,58\%), Pozuelo de Alarcón (4.465 empleos, 3,4\%), Majadahonda (3.781 empleos, 2,9\%) o Tres Cantos (3.462 empleos, $2,6 \%$ ), entre otros. Todos ellos presentan una elevada especialización a nivel regional (salvo en el caso de Alcobendas), destacando muy por encima del resto el caso de Boadilla del Monte, sede de la ciudad financiera del Banco Santander y, a bastante distancia, el de Tres Cantos, en relación con su Parque Tecnológico. Los niveles de empleo y especialización por distritos no difieren mucho del cluster anterior, salvo por el aumento de la importancia de las actividades en el entorno de Barajas. Igualmente, cabe aludir a la localización en el distrito de Fuencarral- El Pardo de la ciudad financiera del BBVA o del Distrito C de Telefónica.

Por último, el mayor volumen de trabajadores en industrias culturales se localiza en Madrid (34.563 empleos, $64,5 \%$ del total). No obstante, un notable volumen de empleo se distribuye por el resto de la región metropolitana, donde en este caso destacan Pozuelo de Alarcón (7.930 empleos, 14,81\%), San Sebastián de los Reyes (1.585 empleos, 4,6\%) y las Rozas de Madrid (1.475 empleos, 2,7\%). En todo caso, el nivel de especialización del empleo en las industrias culturales resulta especialmente elevado en algunos núcleos, como por ejemplo Pozuelo de Alarcón o San Sebastián de los Reyes, en relación con la Ciudad de la Imagen o los estudios de Atresmedia respectivamente; pero también en buena parte del resto de la región metropolitana, incluidos sectores de la sierra madrileña. Por otro lado, dentro del municipio de Madrid, destacan especialmente los distritos de Centro, Hortaleza, San Blas-Canillejas y Fuencarral-El Pardo, con niveles superiores a 3.000 empleos en industrias culturales y elevados niveles de especialización en el contexto regional.

En definitiva, nuestros resultados apuntan a la desigual importancia de los clusters innovadores en la economía de la región de Madrid. Por un lado, se confirma el papel de la ciudad como localización prefe- 
Cluster 1,

de Industrias de alta intensidad tecnológica

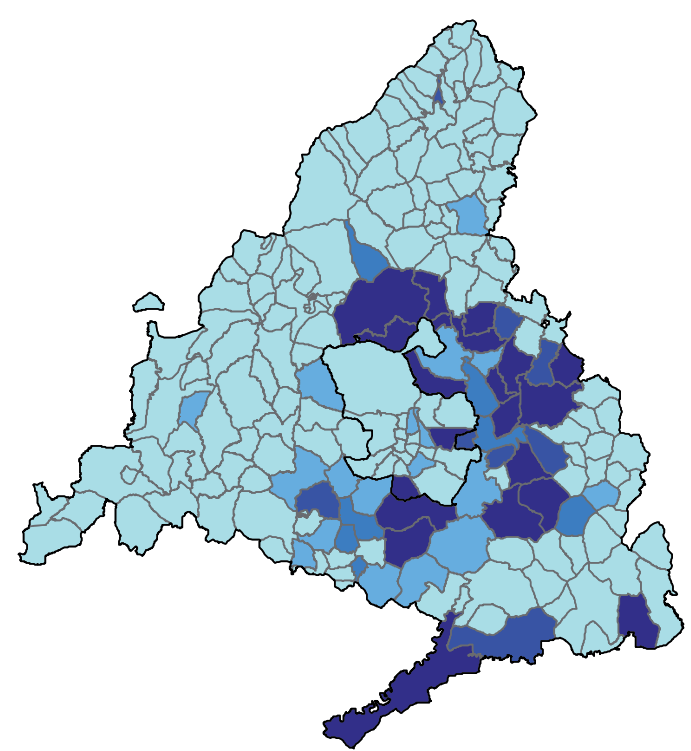

Cluster 3,

de Otros servicios intensivos en conocimiento

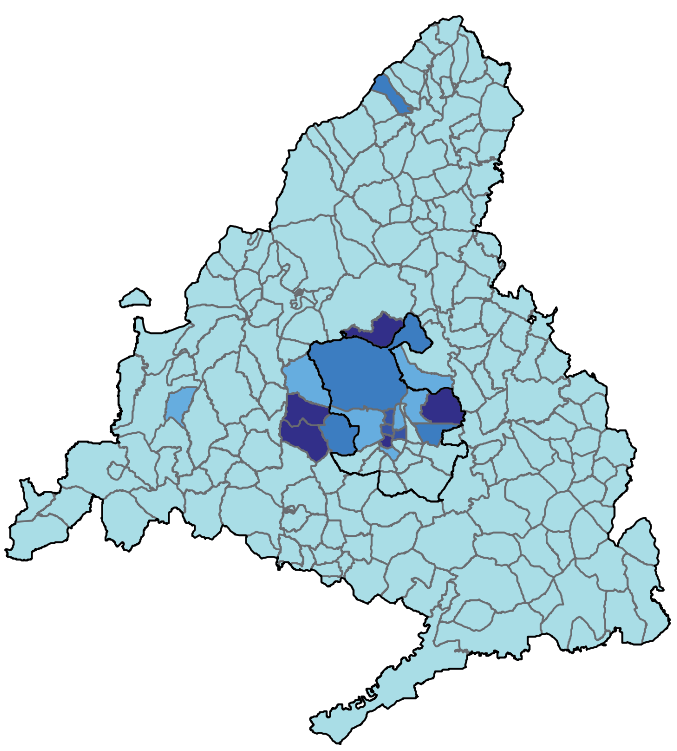

Cluster 2,

de Servicios avanzados a las empresas

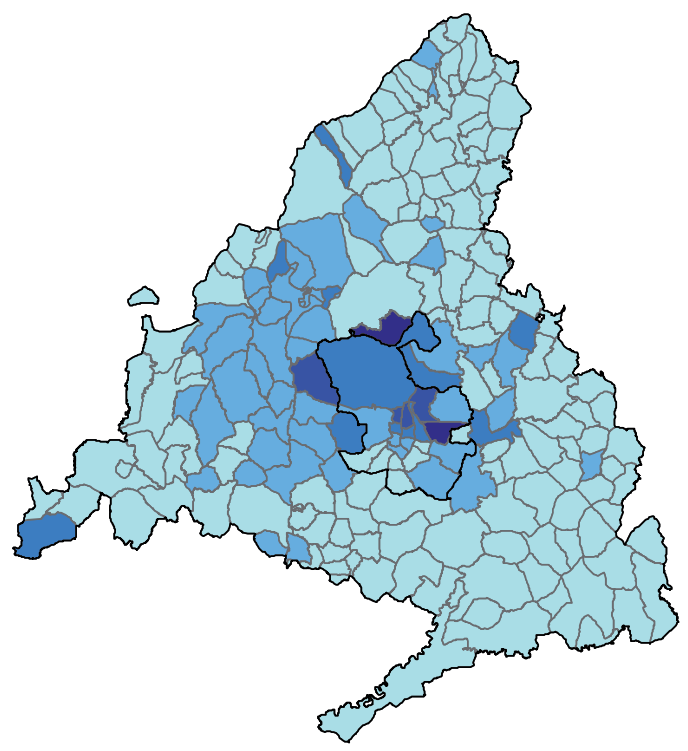

Cluster 4, de Industrias culturales

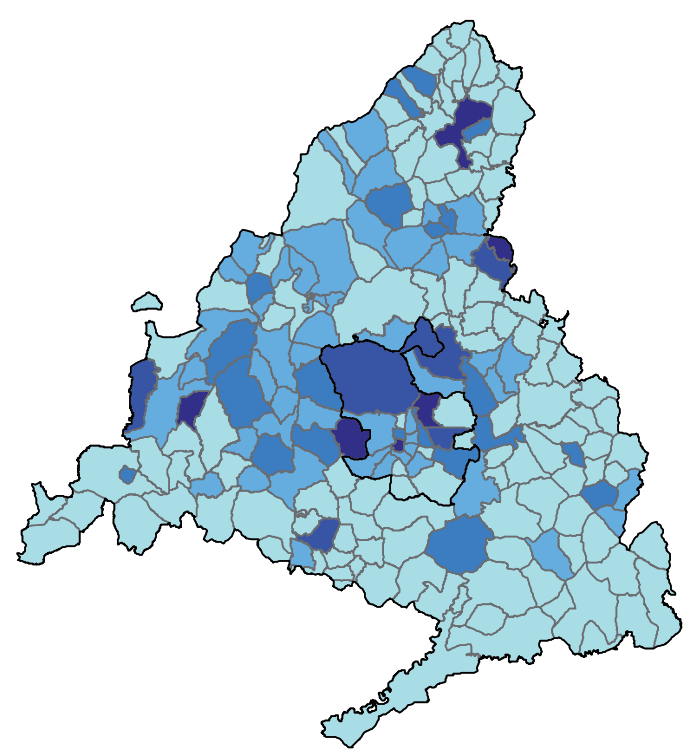

$\underline{156}$

Cociente de localización
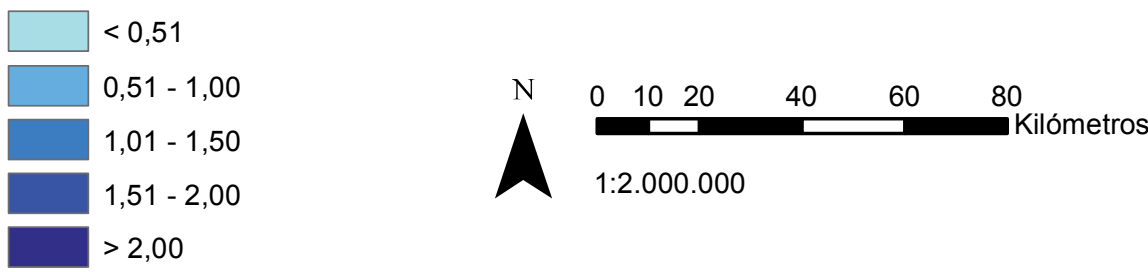

$1: 2.000 .000$

Figura 1. Localización del empleo según clusters innovadores en la Comunidad de Madrid (2016). Fuente: Elaboración propia a partir de Seguridad Social (2016). 
rente de las actividades del terciario avanzado. Destaca así el volumen de actividad en servicios avanzados y empresas financieras nacionales e internacionales, que aportarían al resto del tejido empresarial un conocimiento estratégico para la inserción de Madrid en la globalización y que encuentran en la ciudad ventajas de localización relacionadas con la generación de economías de urbanización y de red, sin olvidar otros factores relacionados con la localización en zonas de prestigio (Taylor, P.J., 2015; Sánchez Moral, S. et al., 2008). No obstante, como hemos comprobado, la influencia de algunos de estos factores no se limita a la ciudad central. Por otro lado, se confirma el atractivo de esta última para las industrias culturales, en relación sobre todo con la densidad de redes empresariales del sector o el valor simbólico de su centro histórico-cultural (Méndez, R. et al., 2010). Frente a esto, resulta evidente el impacto en las últimas décadas de los procesos de descentralización productiva y deslocalización de la industria de alta intensidad tecnológica hacia el resto de la región metropolitana (Sánchez Moral, S., 2009; Rama, R., 2007).

\section{CONCLUSIONES}

Los resultados obtenidos permiten orientar la discusión sobre el carácter estratégico de los clústeres innovadores para la región de Madrid, incluida la ciudad central. En ese sentido, cabe recordar que de acuerdo con autores como W. Wu (2005), A.J. Scott (2009) o S. Musterd y A. Murie (2010) la desigual dotación de los inputs necesarios para el desarrollo de las actividades intensivas en conocimiento, incluidas las economías de aglomeración, junto con otros factores más relacionados con el atractivo urbano que influirían sobre las localización de los trabajadores, explicarían la tendencia a la concentración de estas actividades en los principales núcleos urbanos. Las estrategias y políticas pueden jugar un papel importante en el impulso de estos procesos.

De este modo, estas tendencias (visibles en el caso de Madrid) se ven hoy en día reforzadas por las directrices planteadas desde diferentes instituciones internacionales y organismos multilaterales de cooperación económica (UNCTAD, Banco Mundial, UE, OCDE). Muchos de ellos aluden constantemente a la necesidad de favorecer una economía más intensiva en conocimiento e innovación, como vehículo para hacer frente no sólo al reto de la competitividad (resuelto con frecuencia a favor de aquellas regiones capaces de asegurar ventajas competitivas dinámicas, es decir, basadas en la innovación), sino también de la sostenibilidad y la equidad. De este modo, se entiende que con ello se puede ayudar a mitigar cuestiones socioeconómicas muy acuciantes como el envejecimiento, la desigualdad o la transición hacia una economía eficiente en el uso de recursos y baja en carbono (Comisión Europea, 2010a).

En la presente investigación hemos podido comprobar cómo esta visión se repite a escala regional, donde es bien conocido el esfuerzo realizado en la última década en relación con las estrategias de especialización inteligente desarrolladas en el marco de la nueva Política Regional y de Cohesión de la UE, pero también entre las grandes ciudades europeas competidoras de Madrid. Resulta así común la identificación de un núcleo de actividades intensivas en tecnología y conocimiento, incluidas algunas de naturaleza servindustrial, como pueden ser las TIC, las telecomunicaciones o las industrias creativas. No obstante, cabe recordar la frecuente consideración de otras actividades como el turismo, la educación y la energía (algunas con importantes implicaciones para la competitividad urbana). Todo ello plantea una primera reflexión sobre el alcance de esas visiones complementarias de la economía del conocimiento -sectorial y transversal-y su reflejo en las políticas y estrategias de promoción económica o de innovación.

En ese sentido, pese a tratarse de sectores de opción preferente a la hora de desarrollar políticas productivas de orden sectorial, los clusters innovadores tienen un comportamiento muy heterogéneo en el caso de Madrid, desde el punto de vista de sus dinámicas temporales y espaciales. Como hemos comprado, las estrategias desplegadas por las empresas en un mundo cada vez más globalizado han tenido un impacto desigual en Madrid, reforzando en algunos casos los procesos deslocalización en la región iniciados hace décadas. También ha sido desigual el impacto de la crisis internacional y las políticas de ajuste posteriores (Méndez, R., et al., 2015). Al respecto, algunos autores ya han señalado los impactos dentro del sistema regional de innovación, en términos por ejemplo del desempeño innovador de las empresas o del esfuerzo en ciencia y tecnología (ver por ejemplo Jordá, R., et al., 2014). 
Nuestra aproximación a través del empleo confirma que los clusters innovadores juegan hoy un papel importante desde el punto de vista de su provisión en la región de Madrid y, especialmente, en la ciudad central, donde su peso supera ya la cuarta parte del total del empleo. Son además fundamentales para la articulación interna del sistema productivo y del sistema regional de innovación, asegurando además la conexión con la economía global. Todo ello, junto con la capacidad de resiliencia exhibida por varias de estas actividades (muy especialmente de los servicios avanzados a las empresas y las industrias culturales y creativas), hace que puedan ser consideradas estratégicas de cara a articular nuevos programas de desarrollo urbano y regional. En cualquier caso, cabe concluir que la opción política y de planificación de una determinada ciudad o región para potenciar económicamente los territorios, no puede solo circunscribirse a decisiones tomadas desde una perspectiva sectorial. Como se viene señalando, ello requiere también de aproximaciones transversales, siendo importante mejorar la gobernanza y asegurar una política coherente, garantizando la coordinación entre los actores regionales, nacionales e internacionales además de proporcionar condiciones adecuadas a las personas e instituciones para innovar. Al mismo tiempo, todo ello debe conducir a resolver los retos sociales que se presentan en la actualidad (OCDE, 2010b, p3).

\section{REFERENCIAS}

Boix, R. (2012). Facing globalization and increased trade: Catalonia's evolution from industrial region to knowledge and creative economy. Regional Science Policy and Practice, 4, 97-112. doi: https://doi.org/10.1111/j.17577802.2011.01057.x

Collado, J.C., et al. (2003). La economía de Madrid según la tabla input-output de 2000. Madrid: Comunidad de Madrid.

Comisión Europea (2010a). Europa 2020: Una estrategia para un crecimiento inteligente, sostenible e integrador. Bruselas: COM, Comisión Europea.

Comisión Europea (2010b). Contribución de la Política Regional al crecimiento inteligente en el marco de Europa 2020. Bruselas: COM, Comisión Europea.

Comisión Europea (2010c). Libro Verde. Liberar el potencial de las industrias culturales y creativas. Bruselas: COM, Comisión Europea.

Comisión Europea (2013): Octavo informe de situación sobre la cohesión económica, social y territorial. La dimensión regional y urbana de la crisis. Bruselas: Comisión Europea. Recuperado de http://eur-lex.europa.eu/LexUriServ/LexUriServ. do?uri=COM:2013:0463:FIN:ES:PDF. Último acceso: 1 de junio de 2014.

Comunidad de Madrid (2013): Estrategia Regional de Investigación e Innovación 2014-2020 (RIS3). Madrid: Comunidad de Madrid. [En línea]. Recuperado de http://www.comunidad.madrid/sites/default/files/estrategia_ris_3_madrid_ v21.2.pdf. Último acceso: 1 de enero de 2016.

Cooke, P. \& Lazzeretti, L. (2008). Creative Cities, Cultural Clusters and Local Economic Development. Cheltenham: Edward Elgar. doi: https://doi.org/10.4337/9781847209948

Cuadrado-Roura, J.R. (2013). Service Industries and Regions - Growth, Location and Regional Effects. Berlin-Heidelberg: Springer-Verlag. https://doi.org/10.1007/978-3-642-35801-2

DCMS (1998). Creative Industries Mapping Document. London: DCMS (Department for Culture, Media and Sport).

ESPON- Politécnico de Milano (2013). KIT ESPON: Knowledge, Innovation and Territory. Recuperado de https://www.espon. eu/sites/default/files/attachments/KIT_Interim-Report.pdf Último acceso: 26 de abril de 2019].

Foray, D.; David, P.A. \& Hall, B.H. (2011). Smart specialization. From academic idea to political instrument, the surprising career of a concept and the difficulties involved in its implementation, MTEI-Working Paper, Lausanne: Management of Technology \& Entrepreneurship Institute. Recuperado de https://pdfs.semanticscholar.org/29ad/6773ef30f362d7d3937c483003d974bc91c5.pdf. Último acceso: 1 de enero de 2016.

Glaeser, E.L. (2011). Triumph of the city: how our greatest invention makes us richer, smarter, greener, healthier, and happier. New York: Penguin Press.

Jordá, R., Ruiz, F., González, R. y Contreras, G. (2014). Caracterización del Sistema Nacional de Innovación español (SNI) y de los SRIs durante la crisis. En Albertos J. M. y Sánchez, J. L. (Coords.) Geografía de la crisis económica en España (pp. 263-304). Valencia: Universidad de Valencia. 
Machlup, F. (1962). The production and distribution of knowledge in the United States. Princeton: Princeton University Press. Méndez, R. (2013a). Economía del conocimiento y nuevos contrastes territoriales en España: una perspectiva multiescalar. Boletín de la Asociación de Geógrafos Españoles, 63, 7-32.

Méndez, R., Ondátegui, J. y Sánchez Moral, S. (2007): La estructura territorial de las actividades económicas y la renta. En J.L. García Delgado (Dir.). Estructura económica de Madrid. (pp. 137-183). Madrid: Thomson Civitas.

Méndez, R., y Sánchez, S. (2011). Spanish cities in the knowledge economy: Theoretical debates and empirical evidence. European Urban and Regional Studies, 18 (2), 136-155. doi: https://doi.org/10.1177/0969776410381039

Méndez, R., Sánchez-Moral, S., y Malfeito, J. (2016). Employment changes in knowledge-based industries in large urban areas of Spain: Impact of the economic crisis and austerity policies. Environment and Planning C: Government and Policy, 34 (5), 963-980. doi: https://doi.org/10.1177/0263774X15614698

Musterd, S., Bontje, M., Chapain, C., Kovács, Z. \& Murie, A. (2007). Accomodating creative knowledge. A literature review from a European perspective. ACRE Report 1. Amsterdam. Netherlands: University of Amsterdam. Recuperado de http:// acre.socsci.uva.nl/results/documents/WP1StateoftheArtversion15mrt2007.pdf Último acceso: 26 de abril de 2019.

Musterd, S. \& Murie, A. (2010). Making competitive cities. Pathways, actors and policies. London, UK: Blackwell.

Navarro Arancegui, M. y Gibaja Martins, J.J. (2009). Las tipologías en los sistemas regionales de innovación. El caso de España. Ekonomiaz, 70, 241-281.

OCDE (2010a). The OCDE Innovation Strategy. Getting a head start on tomorrow. Recuperado de https://read.oecd-ilibrary. org/science-and-technology/the-oecd-innovation-strategy_9789264083479-en\#page1 Último acceso: 26 de abril de 2019]. ISBN 978-92-64-08347-9.

OCDE (2010b). The OCDE Innovation Strategy. Ministerial report on the OECD Innovation Strategy. Innovation to strengthen growth and address global and social challenges. Recuperado de https://www.oecd.org/sti/45326349.pdf Último acceso: 20 de mayo de 2019.

OCDE (2011). Regions and Innovation Policy, OECD Reviews of Regional Innovation. París: OCDE. doi: https://doi.org/10.1787/9789264097803-27-en

Paddison, R. \& Hutton, T. (Eds) (2015). Cities and economic change. Restructuring and dislocation in the global metropolis. London: SAGE Publications.

Pavitt, K. (1984). Sectorial patterns of technical change: Towards a taxonomy and a theory. Research Policy, 13, $343-373$. doi: https://doi.org/10.1016/0048-7333(84)90018-0

Peck, J. (2012). Recreative city: Amsterdam, vehicular ideas and the adaptive spaces of creativity policy. International Journal of Urban and Regional Research, 36 (3), 462-485. doi: https://doi.org/10.1111/j.1468-2427.2011.01071.x

Perlo, M. (2011). Cities in times of crisis. The response of local governments in light to the global economic crisis: the role of the formation of human capital, urban innovation and strategic planning. IURD Working Papers. Berkeley: University of California. Recuperado de https://iurd.berkeley.edu/wp/2011-01.pdf Último acceso: 1 de abril de 2019.

Pratt, A. C. (2012). A world turned upside down: the cultural economy, cities and the new austerity. En Smart, creative, sustainable, inclusive: territorial development strategies in the age of austerity. London, UK: Regional Studies Association.

Rama, R. (2007). Emerging districts facing structural reform: The Madrid electronics district and the reshaping of the Spanish telecom monopoly. Environment and Planning A, 39 (9), 2207-2231. doi: https://doi.org/10.1068/a38270

Salom, J. y Albertos, J. (2009). Redes socioinstitucionales, estrategias de innovación y desarrollo territorial en España. Valencia: Universitat de València.

Sánchez Moral, S., Calatrava, A., Melero, A., Méndez, R., Michelini, J.J. y Tébar, J. (2008): Sectores servindustriales estratégicos. Observatorio Industrial de Ayuntamiento de Madrid. Madrid: Ayuntamiento de Madrid. doi: https://doi. org/10.1080/00343400801932326

Sánchez Moral, S. (2009) Industrial Clusters and New Firm Creation in the Manufacturing Sector of Madrid's Metropolitan Region. Regional Studies, 43 (7), 949-965. doi: http://dx.doi.org/10.1080/00343400801932326

Sánchez-Moral, S. (2015). Transformación productiva, equidad y redes. Revista de Estudios Andaluces 32, 49-75. doi: http:// dx.doi.org/10.12795/rea.2015.i32.03

Scott, A.J. (2008). Social Economy of the Metropolis: Cognitive-Cultural Capitalism and the Global Resurgence of Cities. Oxford: Oxford University Press. doi: https://doi.org/10.1093/acprof:oso/9780199549306.001.0001

Scott, A.J. (2009). Human capital resources and requirements across the metropolitan hierarchy of the USA. Journal of Economic Geography, 9, 207-226. doi: https://doi.org/10.1093/jeg/lbn051 
Taylor, P.J., Hoyler, M. y Sánchez Moral, S. (2013). European cities in globalization: a comparative analysis based on the location strategies of advanced producer services. En Cuadrado Roura, J.R. (Ed.): Service Industries and Regions: Growth, Location and Regional Effects. (pp. 285-304). Berlín: Springer-Verlag. doi: https://doi.org/10.1007/978-3-64235801-2_12

UNESCO (2006). Understanding creative industries: cultural statistics for public-policy making. Paris: UNESCO.

Vanhove, N. (2011). The Economics of Tourism Destinations. London: Elsevier. doi: https://doi.org/10.4324/9780080969978 Warwick, K. (2013). Beyond Industrial Policy. Emerging issues and new trends. Paris: OCDE.

Winden, W., Berg, L., Van D. \& Pol, P. (2007). European cities in the knowledge economy: towards a typology. Urban Studies, 44 (3), 525-549. doi: https://doi.org/10.1080/00420980601131886

Wu, W. (2005). Dynamic Cities and Creative Clusters. World Bank Policy Research Working Paper 3509, 1-35. doi: https://doi. org/10.1596/1813-9450-3509 
Anexo I. Coeficientes técnicos y de la matriz inversa de Leontief de las actividades relacionadas con la economía del conocimiento.

\begin{tabular}{|c|c|c|c|c|c|c|c|c|}
\hline & \multicolumn{2}{|c|}{$\begin{array}{c}\text { Eslabona-mien- } \\
\text { to directo hacia } \\
\text { atrás }\end{array}$} & \multicolumn{2}{|c|}{$\begin{array}{l}\text { Eslabona-mien- } \\
\text { to directo hacia } \\
\text { delante }\end{array}$} & \multicolumn{2}{|c|}{ Efecto de arrastre } & \multicolumn{2}{|c|}{ Efecto empuje } \\
\hline & Int. & Total & Int. & Total & Int. & Total & Int. & Total \\
\hline Maquinaria industrial & 0,170 & 0,630 & 0,315 & 2,007 & 1,228 & 2,692 & 1,137 & 2,331 \\
\hline Material electrónico & 0,140 & 0,813 & 0,001 & 1,292 & 1,180 & 3,115 & 1,000 & 2,217 \\
\hline $\begin{array}{l}\text { Ordenadores y maquinaria de } \\
\text { precisión }\end{array}$ & 0,291 & 0,700 & 0,011 & 0,781 & 1,407 & 2,802 & 1,006 & 1,504 \\
\hline Vehículos y sus piezas & 0,098 & 0,823 & 0,079 & 0,592 & 1,122 & 3,421 & 1,096 & 2,220 \\
\hline Otro material de transporte & 0,134 & 0,642 & 0,266 & 0,728 & 1,180 & 2,731 & 1,145 & 1,795 \\
\hline Productos farmacéuticos & 0,133 & 0,598 & 0,111 & 0,876 & 1,184 & 2,539 & 1,055 & 1,628 \\
\hline Química de base e industrial & 0,185 & 0,712 & 0,145 & 1,670 & 1,256 & 2,842 & 1,135 & 4,432 \\
\hline Informática & 0,412 & 0,632 & 0,353 & 0,378 & 1,629 & 2,531 & 1,904 & 2,398 \\
\hline Servicios de información & 0,412 & 0,613 & 0,422 & 0,525 & 1,629 & 2,455 & 1,251 & 1,404 \\
\hline $\begin{array}{l}\text { Actividades jurídicas y de } \\
\text { contabilidad }\end{array}$ & 0,367 & 0,449 & 0,483 & 0,723 & 1,560 & 1,955 & 1,879 & 2,850 \\
\hline $\begin{array}{l}\text { Sedes centrales y gestión } \\
\text { empresarial }\end{array}$ & 0,453 & 0,600 & 0,460 & 0,543 & 1,733 & 2,373 & 1,757 & 2,214 \\
\hline Servicios técnicos & 0,310 & 0,571 & 0,305 & 0,405 & 1,434 & 2,365 & 1,547 & 2,103 \\
\hline Publicidad y estudios de mercado & 0,423 & 0,651 & 0,667 & 0,865 & 1,648 & 2,732 & 2,239 & 3,347 \\
\hline Otras actividades profesionales & 0,280 & 0,517 & 0,056 & 0,517 & 1,402 & 2,246 & 1,082 & 2,224 \\
\hline Telecomunicaciones & 0,268 & 0,577 & 0,228 & 0,382 & 1,383 & 2,381 & 1,967 & 3,330 \\
\hline Intermediación financiera & 0,100 & 0,145 & 0,440 & 0,674 & 1,137 & 1,283 & 1,823 & 2,751 \\
\hline Seguros y fondos de pensiones & 0,327 & 0,640 & 0,271 & 0,387 & 1,412 & 1,853 & 1,213 & 1,507 \\
\hline Auxiliares financieros y de seguros & 0,112 & 0,172 & 0,483 & 0,767 & 1,150 & 1,318 & 1,314 & 1,655 \\
\hline Edición & 0,472 & 0,701 & 0,364 & 0,832 & 1,689 & 2,780 & 1,248 & 1,778 \\
\hline Cine, video, TV, radio y sonido & 0,422 & 0,765 & 0,619 & 1,057 & 1,709 & 3,463 & 2,038 & 4,296 \\
\hline Servicios recreativos & 0,410 & 0,483 & 0,080 & 0,166 & 1,589 & 2,055 & 1,088 & 1,305 \\
\hline
\end{tabular}

Nota: Int.: Valores interiores; Total: valores totales (incluye importación).

Fuente: Elaboración propia, procedente y adaptado de Ayuntamiento de Madrid (2016). 